\title{
Contact Pseudo-slant submanifolds of a cosymplectic manifold
}

\author{
Mehmet Atceken ${ }^{1}$, Umit Yildirim ${ }^{1}$ and Suleyman Dirik ${ }^{2}$ \\ ${ }^{1}$ Department of Mathematics, University of Gaziosmanpasa, Tokat, Turkey \\ ${ }^{2}$ Department of Mathematics, University of Amasya, Amasya, Turkey
}

Received: 30 May 2018, Accepted: 4 November 2018

Published online: 29 December 2018.

\begin{abstract}
This paper is concerned with the study of the contact pseudo-slant submanifolds of a cosymplectic manifold. We derive the integrability conditions of involved distributions in the definition of a pseudo-slant submanifold. The notion contact parallel and contact pseudo-slant product is defined and the necessary and sufficient conditions for a submanifold to be contact parallel and contact pseudo-slant product are given. Also, an non-trivial example is used to demonstrate that the method presented in this paper is effective.
\end{abstract}

Keywords: Cosymplectic manifold, cosymplectic space form, contact slant submanifold, contact pseudo-slant submanifold.

\section{Introduction}

B-Y. Chen introduced the concept of slant submanifold through differential points of view as a generalization of complex and totally real submanifold of an almost Hermitian manifold[2]. After then, Papaghuic initiated the notion of semi-slant submanifolds as a generalization of slant submanifolds and CR-submanifolds[3].

Furthermore, Carriazo defined pseudo-slant submanifold with the name anti-slant submanifolds as a special class of bi-slant submanifolds[4]. Also pseudo-slant submanifolds have been studied by Khan et. al. in [5]. Later, U. C. De et. al. studied and characterized pseudo-slant submanifolds of trans-Sasakian Manifolds[6]

Recently, M. Atceken and S. Dirik also have investigated contact pseudo-slant submanifolds in cosymplectic space forms and gave some results om mixed-geodesic, totally geodesic and the induced tensor fields to be parallel[7].

\section{Preliminaries}

An odd-dimensional counterpart of a Kaehler manifold is given by a cosymplectic manifold, which is locally a product of a Kaehler manifold with a circle or a line.

A $(2 n+1)$-dimensional $C^{\infty}$-manifold $\bar{M}$ is said to be have an almost contact structure if there exist on $\bar{M}$ a tensor field $\varphi$ of type $(1,1)$, a vector field $\xi$ and 1 -form $\eta$ satisfying;

$$
\varphi^{2}=-I+\eta \oplus \xi, \varphi \xi=0, \eta o \varphi=0, \eta(\xi)=1
$$


There always exists a Riemannian metric $g$ on an almost contact manifold $\bar{M}$ satisfying the following compatibility condition

$$
g(\varphi X, \varphi Y)=g(X, Y)-\eta(X) \eta(Y), \eta(X)=g(X, \xi)
$$

where $X$ and $Y$ are vector fields on $\bar{M}$.

An almost contact structure $(\varphi, \xi, \eta)$ is said to be normal the almost complex $J$ on the product manifold $\bar{M} x \mathbb{R}$ given by

$$
J\left(X, f \frac{d}{d t}\right)=\left(\varphi X-f \xi, \eta(X) \frac{d}{d t}\right)
$$

where $f$ is a differentiable function on $\bar{M} x \mathbb{R}$, has no torsion, i.e., $J$ is integrable. The condition for normality in terms of $\varphi, \xi, \eta$ is $[\varphi, \varphi]+2 d \eta \oplus \xi=0$ on $\bar{M}$, where $[\varphi, \varphi]$ is the Nijenhuis tensor of $\varphi$. Finally the fundamental 2 -form $\Phi$ is defined by $g(X, \varphi Y)=\Phi(X, Y)$. Al almost contact metric structure $(\varphi, \xi, \eta, g)$ is said to be cosymplectic structure if it is normal and $\Phi$ and $\eta$ are closed, that is,

$$
\left(\bar{\nabla}_{X} \varphi\right) Y=0
$$

for any $X, Y \in \Gamma(T \bar{M})$, where $\Gamma(T \bar{M})$ is the set of the differentiable vector fields on $\bar{M}$ and $\bar{\nabla}$ denotes the Levi-Civita connection on $\bar{M}[8]$.

A plane section $\pi$ in $T_{\bar{M}}(p)$ of an almost contact metric manifold $\bar{M}$ is called a $\varphi$-section if $\pi \perp \xi$ and $\varphi(\pi)=\pi$. $M$ is of constant $\varphi$-sectional curvature if sectional curvature $K(\pi)$ does not depend on the choice of the $\varphi$-section $\pi$ of $T_{\bar{M}}(p)$ and the choice of a point $p \in \bar{M}$. A cosymplectic manifold $\bar{M}$ is said to be a cosymplectic space form if the $\varphi$ sectional curvature is constant c along $\bar{M}$. A cosymplectic space form will be denoted by $\bar{M}(c)$. Then the Riemannian curvature tensor $\bar{R}$ on $\bar{M}(c)$ is given by

$$
\begin{aligned}
\bar{R}(X, Y, Z, W) & =\frac{c}{4}\{g(X, W) g(Y, Z)-g(X, Z) g(Y, W)+g(X, \varphi W) g(Y, \varphi Z)-g(X, \varphi Z) g(Y, \varphi W)-2 g(X, \varphi Y) g(Z, \varphi W) \\
& -g(X, W) \eta(Y) \eta(Z)+g(X, Z) \eta(Y) \eta(W)-g(Y, Z) \eta(X) \eta(W)+g(Y, W) \eta(X) \eta(Z)\}
\end{aligned}
$$

for any $X, Y, Z, W \in \Gamma(T \bar{M})$.

Now, let $M$ be a submanifold of an almost contact metric manifold $\bar{M}$, we denote the induced connections on $M$ and the normal bundle $T^{\perp} M$ by $\nabla$ and $\nabla^{\perp}$, respectively, then the Gauss and Weingarten formulas are given by

$$
\bar{\nabla}_{X} Y=\nabla_{X} Y+h(X, Y)
$$

and

$$
\bar{\nabla}_{X} V=-A_{V} X+\nabla_{X}^{\perp} V
$$

for any $X, Y \in \Gamma(T M), V \in \Gamma\left(T^{\perp} M\right)$, where $h$ is the second fundamental form and $A_{V}$ is the Weingarten map associated with $V$ as

$$
g\left(A_{V} X, Y\right)=g(h(X, Y), V)
$$


We denote the Riemannian curvature tensor of $M$ by $R$, then the Gauss equation and Weingarten formulas imply

$$
\bar{R}(X, Y) Z=R(X, Y) Z-A_{h(Y, Z)} X+A_{h(X, Z)} Y+\left(\nabla_{X} h\right)(Y, Z)-\left(\nabla_{Y} h\right)(X, Z),
$$

for any $X, Y, Z \in \Gamma(T M)$. Taking the normal component of (9), we reach at equation of Codazzi

$$
(\bar{R}(X, Y) Z)^{\perp}=\left(\nabla_{X} h\right)(Y, Z)-\left(\nabla_{Y} h\right)(X, Z) .
$$

If $(\bar{R}(X, Y) Z)^{\perp}=0$, then submanifold is said to be curvature-invariant.

Next we define the curvature tensor $R^{\perp}$ of the normal bundle of $M$ by

$$
g\left(R^{\perp}(X, Y) V, U\right)=g(\bar{R}(X, Y) U, V)-g\left(\left[A_{V}, A_{U}\right] X, Y\right),
$$

for any $X, Y \in \Gamma(T M)$ and $U, V \in \Gamma\left(T^{\perp} M\right)$. If $R^{\perp}=0$, then normal connection of $M$ is said to be flat.

Definition 1.If the normal curvature tensor $R^{\perp}$ of $M$ satisfies

$$
R^{\perp}(X, Y) V=2 g(f X, Y) V
$$

for any $X, Y \in\left(\varphi^{2} \Gamma(T M)\right)$ and $V \in \Gamma\left(T^{\perp} M\right)$, then the normal connection of $M$ is said to be contact flat.

Furthermore, for any $X \in \Gamma(T M)$, we can write

$$
\varphi X=f X+\omega X,
$$

where $f X$ and $\omega X$ denote the tangential and normal components of $\varphi X$, respectively. Similarly, for $V \in \Gamma\left(T^{\perp} M\right), \varphi V$ also can be written

$$
\varphi V=B V+C V,
$$

where $B V$ and $C V$ denote, respectively, the tangential and normal components of $\varphi V$. By using (1), (12), (13) and taking into account of $\xi$ being tangent to $M$, we get

$$
f^{2}+B \omega=-I+\eta \otimes \xi, \omega f+C \omega=0,
$$

and

$$
f B+B C=0, \omega B+C^{2}=-I .
$$

Here the covariant derivations of tensor fields $f, \omega, B$ and $C$ are defined by

$$
\begin{gathered}
\left(\nabla_{X} f\right) Y=\nabla_{X} f Y-f \nabla_{X} Y, \\
\left(\bar{\nabla}_{X} \omega\right) Y=\nabla_{X}{ }^{\perp} \omega Y-\omega \nabla_{X}{ }^{\perp} Y, \\
\left(\bar{\nabla}_{X} B\right) V=\nabla_{X} B V-B \nabla_{X}^{\perp} V, \\
\left(\bar{\nabla}_{X} C\right) Y=\nabla_{X}^{\perp} C V-C \nabla_{X}^{\perp} V,
\end{gathered}
$$


for any $X, Y \in \Gamma(T M)$ and $V \in \Gamma\left(T^{\perp} M\right)$. By using (4), (6), (7) and (12), we can easily to see that

$$
\begin{gathered}
\left(\nabla_{X} f\right) Y=A_{\omega Y} X+B h(X, Y), \\
\left(\nabla_{X} \omega\right) Y=-h(X, f Y)+C h(X, Y), \\
\left(\nabla_{X} C\right) V=-\omega A_{V} X-h(X, B V)
\end{gathered}
$$

and

$$
\left(\nabla_{X} B\right) V=A_{C V} X-f A_{V} X
$$

for any $X, Y \in \Gamma(T M)$ and $V \in \Gamma\left(T^{\perp} M\right)$. By using (21) and (23), we can easily to see that

$$
g\left(\left(\nabla_{X} \omega\right) Y, V\right)=-g\left(\left(\nabla_{X} B\right) V, Y\right)
$$

\section{Contact slant submanifolds of a cosymplectic manifold}

Let $\mathrm{M}$ be a submanifold of an almost contact metric manifold $(\bar{M}, \varphi, \xi, \eta, g)$. Then $\mathrm{M}$ is said to be a contact slant submanifold if the angle $\theta(X)$ between $\varphi X$ and $T_{M}(p)$ is constant at any point $p \in M$ for any $X$ linearly independent of $\xi$. Thus the invariant and anti-invariant submanifolds are special class of slant submanifolds with slant angles $\theta=0$ and $\theta=\frac{\pi}{2}$, respectively. If the slant angle $\theta$ is neither zero nor $\frac{\pi}{2}$, then slant submanifold is said to be proper contact slant submanifold. The slant submanifolds of an almost contact metric manifold, the following theorem is well known.

Theorem 1. Let $M$ be a submanifold of an almost contact metric manifold $\bar{M}$ such that $\xi \in \Gamma(T M)$. M is a contact slant submanifold if and only if there exists a constant $\lambda \in(0,1)$ such that

$$
f^{2}=\lambda(-I+\eta \otimes \xi)
$$

Furthermore, if $\theta$ is slant angle of $M$, then it satisfies $\lambda=\cos ^{2} \theta$.

As a consequence of the above Theorem and (14), we have the following relations;

$$
\begin{aligned}
g(f X, f Y) & =\cos ^{2} \theta\{g(X, Y)-\eta(X) \eta(Y)\} \\
g(\omega X, \omega Y) & =\sin ^{2} \theta\{g(X, Y)-\eta(X) \eta(Y)\}, \\
B \omega & =\sin ^{2} \theta(-I+\eta \otimes \xi) .
\end{aligned}
$$

For a slant submanifold $M$ of an almost contact metric manifold $\bar{M}$, the normal bundle $T^{\perp} M$ of $M$ is decomposable as

$$
T^{\perp} M=\omega(T M) \oplus \mu
$$

where $\mu$ is the invariant normal subbundle with respect to $\varphi$. 


\section{Contact Pseudo-slant submanifolds in cosymplectic manifold}

Definition 2. Let $M$ be a submanifold of a cosymplectic manifold $\bar{M}(\varphi, \xi, \eta, g)$. We say that $M$ is a contact pseudo-slant submanifold if there exists a pair of orthogonal distributions $D^{\perp}$ and $D^{\theta}$ on $M$ such that

(i) The distribution $D^{\perp}$ is totally real, i.e., $\varphi\left(D^{\perp}\right) \subseteq T^{\perp} M$,

(ii) The distribution $D^{\theta}$ is slant with slant angle $\theta$,

(iii) The tangent space TM admits the orthogonal direct decomposition $T M=D^{\perp} \oplus D^{\theta}$.

If we denote the dimensions of $D^{\perp}$ and $D^{\theta}$ by $p$ and $q$, respectively, the we have the following possible cases;

(i) if $p=0$, then $M$ is a slant submanifold,

(ii) if $q=0$, then $M$ is an anti-invariant submanifold,

(iii) if $p q \neq 0, \theta=0$, then $M$ is a contact CR-submanifold.

For a pseudo-slant submanifold $M$ of a cosymplectic manifold $\bar{M}$, the normal bundle $T^{\perp} M$ of a pseudo-slant submanifold $M$ is decomposable as

$$
T^{\perp} M=\varphi\left(D^{\perp}\right) \oplus \omega\left(D^{\theta}\right) \oplus \mu, \varphi\left(D^{\perp}\right) \perp \omega\left(D^{\theta}\right) .
$$

Example 1. Let us consider the Euclidean space $\mathbb{R}^{11}$ with the cartesian coordinates $\left(x_{1}, y_{1}, x_{2}, y_{2}, x_{3}, y_{3}, x_{4}, y_{4}, x_{5}, y_{5}, t\right)$ and almost contact metric structure

$$
\begin{aligned}
& \varphi\left(\sum_{i=1}^{5}\left\{X_{i} \frac{\partial}{\partial x_{i}}+Y_{i} \frac{\partial}{\partial y_{i}}\right\}+Z \frac{\partial}{\partial t}\right)=\sum_{i=1}^{5}\left(-Y_{i} \frac{\partial}{\partial x_{i}}+X_{i} \frac{\partial}{\partial x_{i}}\right) \\
& \xi=\frac{\partial}{\partial t}, \quad \eta=d t, g=\eta \otimes \eta+\sum_{i=1}^{5}\left(d x_{i}^{2}+d y_{i}^{2}\right) .
\end{aligned}
$$

It is clear that $\mathbb{R}^{11}$ is a cosymplectic manifold with usual Euclidean metric tensor. Let $M$ be a submanifold of $\mathbb{R}^{11}$ defined by

$$
\chi(u, v, s, t)=(v \cos u,-v \sin u, s \cos u,-s \sin u, v+2 s, 2 v-s,-s \cos u,-s \sin u,-v \cos u,-v \sin u, t)
$$

with non-zero $u, v, s, t$. Then the tangent space of $M$ is spanned by the vector fields

$$
\begin{aligned}
& e_{1}=-v \sin u \frac{\partial}{\partial x_{1}}-v \cos u \frac{\partial}{\partial y_{1}}-s \sin u \frac{\partial}{\partial x_{2}}-s \cos u \frac{\partial}{\partial y_{2}}+s \sin u \frac{\partial}{\partial x_{4}}-s \cos u \frac{\partial}{\partial y_{4}}+v \sin u \frac{\partial}{\partial x_{5}}-v \cos u \frac{\partial}{\partial y_{5}} \\
& e_{2}=\cos u \frac{\partial}{\partial x_{1}}-\sin u \frac{\partial}{\partial y_{1}}+\frac{\partial}{\partial x_{3}}+2 \frac{\partial}{\partial y_{3}}-\cos u \frac{\partial}{\partial x_{5}}-\sin u \frac{\partial}{\partial y_{5}} \\
& e_{3}=\cos u \frac{\partial}{\partial x_{2}}-\sin u \frac{\partial}{\partial y_{2}}+2 \frac{\partial}{\partial x_{3}}-\frac{\partial}{\partial y_{3}}-\cos u \frac{\partial}{\partial x_{4}}-\sin u \frac{\partial}{\partial y_{4}}, \\
& e_{4}=\xi=\frac{d}{d t} .
\end{aligned}
$$


Furthermore, with respect to complex structure of $\mathbb{R}^{11}$, we have

$$
\begin{aligned}
\varphi e_{1} & =v \cos u \frac{\partial}{\partial x_{1}}-v \sin u \frac{\partial}{\partial y_{1}}+s \cos u \frac{\partial}{\partial x_{2}}-s \sin u \frac{\partial}{\partial y_{2}}+s \cos u \frac{\partial}{\partial x_{4}}+s \sin u \frac{\partial}{\partial y_{4}}+v \cos u \frac{\partial}{\partial x_{5}}+v \sin u \frac{\partial}{\partial y_{5}} \\
\varphi e_{2} & =\cos u \frac{\partial}{\partial x_{1}}+\cos u \frac{\partial}{\partial y_{1}}-2 \frac{\partial}{\partial x_{3}}+\frac{\partial}{\partial y_{3}}+\sin u \frac{\partial}{\partial x_{5}}-\cos u \frac{\partial}{\partial y_{5}} \\
\varphi e_{3} & =\sin u \frac{\partial}{\partial x_{2}}+\cos u \frac{\partial}{\partial y_{2}}+\frac{\partial}{\partial x_{3}}-2 \frac{\partial}{\partial y_{3}}+\sin u \frac{\partial}{\partial x_{4}}-\cos u \frac{\partial}{\partial y_{4}} \\
\varphi e_{4} & =0
\end{aligned}
$$

Since $g\left(\varphi e_{1}, e_{2}\right)=g\left(\varphi e_{1}, e_{3}\right)=0, \varphi e_{1}$ is orthogonal to $M$ and

$$
\cos \theta=\frac{g\left(\varphi e_{2}, e_{3}\right)}{\left\|e_{2}\right\|\left\|e_{3}\right\|}=\frac{3}{7}
$$

it is easy to see that $D^{\theta}=S p\left\{e_{2}, e_{3}\right\}$ is a slant distribution and $D^{\perp}=S p\left\{e_{1}\right\}$ is an anti-invariant distribution. Thus $M$ is a 4-dimensional proper contact pseudo-slant submanifold of $\mathbb{R}^{11}$. It is easy to check that the distributions $D^{\perp}$ and $D^{\theta}$ are integrable. We denote the integral manifolds of $D^{\perp}$ and $D^{\theta}$ by $M_{\perp}$ and $M_{\theta}$, we can conclude that $M=M_{\perp} x M_{\theta}$.

Theorem 2. Let $M$ be a contact pseudo-slant submanifold of a cosymplectic manifold $\bar{M}$. Then anti-invariant distribution $D^{\perp}$ is always integrable.

Proof. For any $Y, Z \in \Gamma\left(D^{\perp}\right)$, we have

$$
\bar{\nabla}_{Z} \varphi Y=\varphi \bar{\nabla}_{Z} Y-A_{\omega Y} Z+\nabla_{Z}^{\perp} \omega Y=\omega \nabla_{Z} Y+f \nabla_{Z} Y+B h(Z, Y)+C h(Z, Y),
$$

which implies that

$$
-A_{\omega Y} Z=f \nabla_{Z} Y+B h(Y, Z)
$$

Thus we have

$$
f[Y, Z]=A_{\omega Z} Y-A_{\omega Y} Z
$$

Since the ambient manifold $\bar{M}$ is cosymplectic, we have

$$
\begin{aligned}
g\left(A_{\omega Z} Y-A_{\omega Y} Z, U\right) & =g(h(Y, U), \omega Z)-g(h(Z, U), \omega Y) \\
& =g(h(Y, U), \omega Z)-g\left(\bar{\nabla}_{U} Z, \omega Y\right) \\
& =g(h(Y, U), \omega Z)+g\left(\bar{\nabla}_{U} \varphi Y, Z\right) \\
& =g(h(Y, U), \omega Z)-g\left(\bar{\nabla}_{U} Y, \varphi Z\right) \\
& =g(h(Y, U), \omega Z)-g(h(U, Y), \omega Z)=0,
\end{aligned}
$$

for any $U \in \Gamma(T M)$, that is,

$$
A_{\omega Z} Y=A_{\omega Y} Z
$$

From (31) and (32) we conclude that $f[Y, Z]=0$, i.e., $[Y, Z] \in \Gamma\left(D^{\perp}\right)$. The proof is completes. 
Theorem 3. Let $M$ be contact pseudo-slant submanifold of a cosymplectic manifold $\bar{M}$. Then the slant distribution $D^{\theta}$ is integrable if and only if

$$
g\left(A_{C \omega Y} Z+f A_{\omega Z} Y, X\right)=g\left(A_{C \omega X} Z+f A_{\omega Z} X, Y\right),
$$

for any $X, Y \in \Gamma\left(D^{\theta}\right)$ and $Z \in \Gamma\left(D^{\perp}\right)$.

Proof.By using (6), (7) and (27), we have

$$
\begin{aligned}
g([X, Y], Z) & =g\left(\nabla_{X} Y, Z\right)-g\left(\nabla_{Y} X, Z\right) \\
& =g\left(\bar{\nabla}_{Y} Z, X\right)-g\left(\bar{\nabla}_{X} Z, Y\right) \\
& =g\left(\bar{\nabla}_{Y} \varphi Z, \varphi X\right)-g\left(\bar{\nabla}_{X} \varphi Z, \varphi Y\right) \\
& =g\left(\bar{\nabla}_{Y} \varphi Z, f X\right)+g\left(\bar{\nabla}_{Y} \varphi Z, \omega X\right)-g\left(\bar{\nabla}_{X} \varphi Z, f Y\right)-g\left(\bar{\nabla}_{X} \varphi Z, \omega Y\right) \\
& =-g\left(A_{\varphi Z} f X, Y\right)+g\left(A_{\varphi Z} f Y, X\right)+g\left(\bar{\nabla}_{Z} X, \varphi \omega Y\right)-g\left(\bar{\nabla}_{Y} Z, \varphi \omega X\right) \\
& =g\left(A_{\varphi Z} f Y, X\right)-g\left(A_{\varphi Z} f X, Y\right)+g\left(\nabla_{X} Z, B \omega Y\right)-g\left(\nabla_{Y} Z, B \omega X\right)+g\left(\bar{\nabla}_{X} Z, C \omega Y\right)-g\left(\bar{\nabla}_{Y} Z, C \omega X\right) \\
& =g\left(A_{\varphi Z} f Y+f A_{\varphi Z} Y, X\right)-\sin ^{2} \theta g\left(\nabla_{X} Z, Y\right)+\sin ^{2} \theta g\left(\nabla_{Y} Z, X\right)+g(h(X, Z), C \omega Y)-g(h(Y, Z), C \omega X) \\
& =g\left(f A_{\varphi Z} Y+A_{\varphi Z} f Y, X\right)+\sin ^{2} \theta g([X, Y], Z)+g\left(A_{C \omega Y} X-A_{C \omega X} Y, Z\right),
\end{aligned}
$$

for any $X, Y \in \Gamma\left(D^{\theta}\right)$ and $Z \in \Gamma\left(D^{\perp}\right)$. Consequently, we reach at

$$
\cos ^{2} \theta g([X, Y], Z)=g\left(f A_{\varphi Z} Y+A_{\varphi Z} f Y, X\right)+g\left(A_{C \omega Y} X-A_{C \omega X} Y, Z\right),
$$

which proves our assertion.

Theorem 4. Let $M$ be a contact pseudo-slant submanifold of a cosymplectic manifold $\bar{M}$. Then the anti-invariant distribution $D^{\perp}$ defines totally geodesic foliation in $M$ if and only if

$$
A_{\omega Z} f X-A_{\omega f X} Z \in \Gamma\left(D^{\theta}\right)
$$

for any $X \in \Gamma\left(D^{\theta}\right)$ and $Z \in \Gamma\left(D^{\perp}\right)$.

Proof. For any $X \in \Gamma\left(D^{\theta}\right)$ and $Y, Z \in \Gamma\left(D^{\perp}\right)$, we have

$$
\begin{aligned}
g\left(\nabla_{Y} Z, X\right) & =g\left(\bar{\nabla}_{Y} \varphi Z, \varphi X\right)=g\left(\bar{\nabla}_{Y} \varphi Z, \omega X\right)+g\left(\bar{\nabla}_{Y} \varphi Z, f X\right) \\
& =-g\left(A_{\varphi Z} f X, Y\right)-g\left(\bar{\nabla}_{Y} Z, B \omega X\right)-g\left(\bar{\nabla}_{Y} Z, C \omega X\right) \\
& =-g\left(A_{\varphi Z} f X, Y\right)+\sin ^{2} \theta g\left(\nabla_{Y} Z, X-\eta(X) \xi\right)+g\left(A_{\omega f X} Y, Z\right)
\end{aligned}
$$

that is,

$$
\cos ^{2} \theta g\left(\nabla_{Y} Z, X\right)=g\left(A_{\omega f X} Z-A_{\varphi Z} f X, Y\right) .
$$

This proves our assertion.

Theorem 5. Let $M$ be a contact pseudo-slant submanifold of a csoymplectic manifold $\bar{M}$. The slant distribution $D^{\theta}$ defines totally geodesic foliation in $M$ is if and only if

$$
A_{\varphi Z} f Y-A_{\omega f Y} Z \in \Gamma\left(D^{\perp}\right)
$$


for any $Y \in \Gamma\left(D^{\theta}\right)$ and $Z \in \Gamma\left(D^{\perp}\right)$.

Proof. By using, (2), (6) and (7), we have

$$
\begin{aligned}
g\left(\nabla_{X} Y, Z\right) & =-g\left(\bar{\nabla}_{X} Z, Y\right)=-g\left(\nabla_{X} \varphi Z, \varphi Y\right) \\
& =-g\left(\bar{\nabla}_{X} \varphi Z, f Y\right)-g\left(\bar{\nabla}_{X} \varphi Z, \omega Y\right) \\
& =g\left(A_{\varphi Z} f Y, X\right)+g\left(\bar{\nabla}_{X} Z, B \omega Y\right)+g\left(\bar{\nabla}_{X} Z, C \omega Y\right) \\
& =g\left(A_{\varphi Z} f Y, X\right)-\sin ^{2} \theta g\left(\bar{\nabla}_{X} Z, Y-\eta(Y) \xi\right)+g\left(A_{C \omega Y} X, Z\right)
\end{aligned}
$$

for any $X, Y \in \Gamma\left(D^{\theta}\right)$ and $Z \in \Gamma\left(D^{\perp}\right)$. This implies that

$$
\cos ^{2} \theta g\left(\nabla_{X} Y, Z\right)=g\left(A_{\varphi Z} f Y-g\left(A_{C \omega Y} Z, X\right)\right.
$$

Let $M$ be a contact pseudo-slant submanifold of a cosymplectic manifold $\bar{M} . M$ is said to be contact pseudo-slant product if the distributions $D^{\perp}$ and $D^{\theta}$ are totally geodesic in $M$.

From Theorems 4 and 5, we have the following statement.

Proposition 1. Let $M$ be a contact proper pseudo-slant submanifold of a cosymplectic manifold $\bar{M}$. Then $M$ is a contact pseudo-slant product if and only if and only if the shape operator of $M$ satisfies

$$
A_{\varphi D^{\perp}} f\left(D^{\theta}\right)=A_{\omega f D^{\theta}} D^{\perp}
$$

Definition 3. If the second fundamental form $h$ of $M$ satisfies

$$
\left(\bar{\nabla}_{X} h\right)(Y, Z)=g(f X, Y) \omega Z+g(f X, Z) \omega Y,
$$

for any $X, Y, Z \in \varphi^{2}(\Gamma(T M))=\Gamma(T M-\xi)$, then $h$ is said to be contact parallel.

Theorem 6. Let $M$ be a contact pseudo-slant submanifold of a cosymplectic space form $\bar{M}(c)$. If the second fundamental form h of $M$ is contact parallel, then $M$ is either invariant or anti-invariant.

Proof. From (38), we have

$$
\begin{aligned}
\left.\left.\bar{\nabla}_{Y} h\right)(X, Z)-\bar{\nabla}_{X} h\right)(Y, Z) & =g(f Y, X) \omega Z+g(f Y, Z) \omega X-g(f X, Y) \omega Z-g(f X, Z) \omega Y \\
& =2 g(f Y, X) \omega Z+g(f Y, Z) \omega X-g(f X, Z) \omega Y,
\end{aligned}
$$

for any $X, Y, Z \in \Gamma \varphi^{2}(\Gamma M)=\Gamma(T M-\xi)$. Corresponding (5), (9) and (39), we reach at

$$
\left(\frac{c}{4}-1\right)\{2 g(f Y, X) \omega Z+g(f Y, Z) \omega X-g(f X, Z) \omega Y\}=0 .
$$

Setting $Y=Z$ in (40), we conclude that

$$
3\left(\frac{c}{4}-1\right) g(f Y, X) \omega Y=0
$$

which proves our assertion.

Theorem 7. Let $M$ be a proper contact pseudo-slant submanifold of a cosymplectic manifold $\bar{M}$. If the tensor field $B$ is parallel, then $M$ is a contact pseudo-slant product. 
Proof. Since $B$ is parallel, from (23), we have

$$
f A_{\omega Z} U=0, \quad U \in \Gamma(T M), Z \in \Gamma\left(D^{\perp}\right) .
$$

This implies that $A_{\varphi Z} U \in \Gamma\left(D^{\perp}\right)$ and $B h(U, Z)=0$. The proof is completes.

Theorem 8. Let $M$ be a contact pseudo-slant submanifold of a cosymplectic space form $\bar{M}(c)$. $M$ is either anti-invariant submanifold or $\bar{M}$ is flat if $\omega$ is parallel.

Proof. Since $\omega$ is parallel, we can easily to see that

$$
h(f X, Y)=C h(X, Y)=h(X, f Y)
$$

which is equivalent to

$$
f A_{V} X+A_{V} f X=0
$$

for any $X, Y \in \Gamma(T M)$ and $V \in \Gamma\left(T^{\perp} M\right)$. From (41), we have

$$
g\left(A_{V} f X, B U\right)=g\left(A_{V} X, f B U\right)=-g\left(A_{V} X, B C U\right)=-g\left(\omega A_{V} X, C U\right)=0,
$$

for any vector fields $U, V$ normal to $M$. Taking the covariant derivative of (42), for $Y \in \Gamma(T M)$, we obtain

$$
g\left(\bar{\nabla}_{Y} A_{V} f X, B U\right)+g\left(A_{V} f X, \bar{\nabla}_{Y} B U\right)=0 .
$$

This means that

$$
g\left(\left(\nabla_{Y} A\right)_{V} f X+A_{\nabla_{Y}^{\perp} V} f X+A_{V} \nabla_{Y} f X, B U\right)+g\left(A_{V} f X,\left(\nabla_{Y} B\right) U+B \nabla_{Y}^{\perp} U\right)=0 .
$$

Taking into account (24) and (42), we reach at

$$
g\left(\left(\nabla_{Y} A\right)_{V} f X+A_{V}\left\{\left(\nabla_{Y} f\right) X+f \nabla_{Y} X\right\}, B U\right)=0,
$$

from which

$$
g\left(\left(\nabla_{Y} A\right)_{V} f X, B U\right)+g\left(A_{V}\left\{A_{\omega X} Y+B h(X, Y)\right\}, B U\right)=0,
$$

or,

$$
g\left(\left(\nabla_{Y} A\right)_{V} f X, B U\right)+g\left(A_{V} B U, A_{\omega X} Y\right)+g\left(A_{V} B U, B h(X, Y)\right)=0 .
$$

This implies that

$$
g\left(\left(\nabla_{f Y} h\right)(f X, B U), V\right)=g\left(\left(\nabla_{f Y} A\right)_{V} f X, B U\right)=-g\left(A_{V} B U, A_{\omega X} f Y\right)-g\left(A_{V} B U, B h(f Y, X)\right) .
$$


Thus we conclude that

$$
\begin{aligned}
g\left(\left(\nabla_{f X} h\right)(f Y, B U)-\left(\nabla_{f Y} h\right)(f X, B U), V\right) & =g\left(A_{V} B U, A_{\omega X} f Y\right)-g\left(A_{V} B U, A_{\omega Y} f X\right) \\
& =g\left(A_{V} B U, A_{\omega X} f Y+f A_{\omega Y} X\right) \\
& =g\left(A_{V} A_{\omega X} f Y, B U\right)-g\left(A_{V} f A_{\omega Y} X, B U\right) \\
& =g\left(A_{V} f A_{\omega X} Y, B U\right)=0 .
\end{aligned}
$$

On the other hand, form the Codazzi equation, we have

$$
\begin{aligned}
g\left(\left(\nabla_{f X} h\right)(f Y, B U)-\left(\nabla_{f Y} h\right)(f X, B U), V\right) & =\frac{c}{4}\left\{g\left(f^{2} Y, B V\right) g(\omega X, U)-g\left(f^{2} X, B V\right) g(\omega Y, U)+2 g\left(f X, f^{2} Y\right) g(\omega B V, U)\right\} \\
& =-\cos ^{2} \theta \frac{c}{4}\{g(Y, B V) g(\omega X, U)-g(X, B V) g(\omega Y, U)+2 g(f X, Y) g(\omega B V, U)\} .
\end{aligned}
$$

In (44), taking $X, Y \in \Gamma\left(D^{\theta}\right)$ and $U=V=\omega Z \in \Gamma\left(T^{\perp} M\right)$ for $Z \in \Gamma\left(D^{\perp}\right)$, and corresponding (43) and (44), we get

$$
\cos ^{2} \theta \frac{c}{2} g(f X, Y) g(Z, Z)=0
$$

This proves our assertion.

Theorem 9. Let $M$ be a contact pseudo-slant curvature-invariant submanifold of a cosymplectic space form $\bar{M}(c)$. Then $M$ is a either anti-invariant submanifold or $\bar{M}$ is flat space form.

Proof. If $M$ is a contact pseudo-slant curvature-invariant submanifold, then from (5) and (10), we conclude

$$
\frac{c}{4}\{g(\varphi Y, Z) \omega X-g(\varphi X, Z)+2 g(X, \varphi Y) \omega Z\}=0,
$$

for any $X, Y, Z \in \Gamma(T M)$. This implies that

$$
3 \frac{c}{4} g(f Y, X) \omega Y=0,
$$

from which, we obtain

$$
3 \frac{c}{4} \cos ^{2} \theta g\left\{(Y, Y)-\eta^{2}(Y)\right\} \omega Y=0 .
$$

The proof is completes.

Theorem 10. Let $M$ be a invariant submanifold of a cosymplectic space form $\bar{M}(c)$ such that the normal connection of $M$ is contact flat. Then $M$ is totally geodesic submanifold if and only if $\bar{M}$ is flat space.

Proof.

$$
g\left(\left[A_{V}, A_{U}\right] X, Y\right)+g\left(R^{\perp}(X, Y) U, V\right)=\frac{c}{4}\{g(\varphi Y, U) g(\varphi X, V)-g(\varphi X, U) g(\varphi Y, V)+2 g(X, \varphi Y) g(\varphi U, V)\},
$$

for any $X, Y \in \Gamma(T M)$ and $U, V \in \Gamma(T \perp M)$. Since the normal connection is contact flat, we get

$$
g\left(\left[A_{V}, A_{U}\right] X, Y\right)=-\frac{c}{2} g(f X, Y) g(\varphi U, V)-2 g(f X, Y) g(U, V) .
$$

In (45), taking $V=\varphi U$, we reach

$$
g\left(\left[A_{\varphi U}, A_{U}\right] X, Y\right)=-\frac{c}{2} g(f X, Y) g(\varphi U, \varphi U) .
$$


Since $M$ is an invariant submanifold, we can derive

$$
A_{\varphi U} Y=\varphi A_{U} Y=-A_{U} f Y
$$

Thus we have

$$
\begin{aligned}
g\left(A_{\varphi U} A_{U} X-A_{U} A_{\varphi U} X, Y\right) & =g\left(A_{\varphi U} Y, A_{U} X\right)-g\left(A_{U} Y, A_{\varphi U} X\right) \\
& =g\left(\varphi A_{U} Y, A_{U} X\right)-g\left(A_{U} Y, \varphi A_{U} X\right) \\
& =2 g\left(A_{U} Y, A_{U} f X\right) \\
& =-\frac{c}{2} g(f X, Y) g(\varphi U, \varphi U),
\end{aligned}
$$

from which

$$
g\left(A_{U} Y, A_{U} Y\right)=-\frac{c}{4} g(Y, Y) g(U, U) .
$$

Since $g$ is a positive definite, this tell us that $M$ is totally geodesic submanifold if and only if $c=0$.

\section{Competing interests}

The authors declare that they have no competing interests.

\section{Authors' contributions}

All authors have contributed to all parts of the article. All authors read and approved the final manuscript.

\section{References}

[1] Atçeken, M. Contact CR-warped product submanifolds in cosymplectic space forms. Collectanea Math. 62(1), 17-26(2011).

[2] Chen, B-Y. Slant immersion. Bull. Austral. Math. Soc. 41, 135-147(1990).

[3] Papaghuic, N. Semi-Slant Submanifolds of a Kaehlerian Manifold. Ann. Şt. Al. I. Cuza Univ. Iaşi. 40, 55-61(1994).

[4] Carriazo, A. Bi-Slant Immersions. In. Proc. ICARAMS 2000, Kharagpur, India, pp: 88-97(2000).

[5] V. A. Khan, M. A. Khan. Pseudo-Slant Submanifolds of Sasakian Manifold. Indian J. Pure and Appl. Math. 38, 31-42(2007).

[6] U. C. De and A. sarkar. On Pseudo-Slant Submanifolds of Trans-Sasakian Manifolds. Proc. East Acad. Sci., 60, 1-11(2011).

[7] S. Dirik and M. Atceken. Pseudo-Slant Submanifolds in Cosymplectic Space Forms. Acta Math. Sapientiae 8.1, 53-74(2016).

[8] D. E. Blair and D. K. Showers. Almost Contact Manifolds with Killing Structures II. J. Diff. Geom. 9, 577-582(1974). 